\title{
Control de la intervención estructural sobre el área del Teatro Romano de Cádiz
}

\author{
Control of the structural intervention in the area of the Roman Theatre \\ of Cadiz \\ $\underline{\text { P. Pachón }}^{(*)}$, E. Rodríguez-Mayorga ${ }^{(*)}$, A. Cobo ${ }^{(*)}$, E. Yanes ${ }^{(*)}$
}

\section{RESUMEN}

El Teatro Romano de Cádiz (España) es parte de la herencia romana que ha permanecido oculta durante siglos bajo el casco histórico de la ciudad. Se trata del teatro romano más antiguo y de mayores dimensiones localizado en España. En el año 2010, se inició una intervención con el fin de descubrirlo. La intervención consiste en la construcción de una serie de bóvedas de hormigón bajo los edificios construidos sobre el teatro aplicando la técnica de la inyección armada. El control de esta intervención se está realizando por medio de dos técnicas: control topográfico y Análisis Modal Operacional. La metodología seguida para el control de la intervención en el área del teatro romano y los resultados obtenidos de la misma son los principales objetivos del presente artículo.

Palabras clave: Inyección armada; control topográfico; Análisis Modal Operacional (OMA); modelo de elementos finitos.

\section{ABSTRACT}

The Roman Theatre of Cadiz (Spain) is a piece of heritage that has been buried for centuries under the historical town. It is the oldest and biggest Roman theatre in Spain. In 2010, an intervention began in order to dig it up but respecting the monumental landmarks over it. The intervention consisted on the construction of some vaults under the blocks by grout injection. The control of this intervention is being done by two different techniques: topographic control points and Operational Modal Analysis. The methodology that has been followed to control the intervention on this area and the obtained results are the main goals of this paper.

Keywords: Grout injection; topographic control; Operational Modal Analysis (OMA); finite element model.

(*) Universidad de Sevilla (España).

Persona de contacto/Corresponding author: ppachon@us.es (P. Pachón)

Cómo citar este artículo/Citation: Pachón, P., Rodríguez-Mayorga, E., Cobo, A., Yanes, E. (2014). Control de la intervención estructural sobre el área del Teatro Romano de Cádiz. Informes de la Construcción, 66(EXTRA-1): mo03, doi: http://dx.doi.org/10.3989/ic.13.086.

Licencia/License: Salvo indicación contraria, todos los contenidos de la edición electrónica de Informes de la Construcción se distribuyen bajo una licencia de uso y distribución Creative Commons Reconocimiento no Comercial 3.o. España (cc-by-nc). 


\section{INTRODUCCIÓN}

El Teatro Romano de Cádiz se localiza en el barrio del Pópulo, en el centro histórico de la ciudad de Cádiz (Figura 1) y parcialmente cubierto por los edificios de dicho barrio. Es el teatro romano más antiguo de la Península Ibérica, datando su construcción de finales del siglo I a.C. Su importancia no solo radica en su antigüedad sino también en su tamaño, pues el diámetro de su cávea, de $120 \mathrm{~m}$, lo hace ser el edificio de mayores dimensiones de su clase en España. Su aforo sería alrededor de unos 20.000 espectadores, un elevado número en comparación con la población de la ciudad en la época de su construcción, aproximadamente 50.000 habitantes. En el siglo III d.C. el teatro fue abandonado, y su estructura, construida con piedra y hormigón, fue usada como base para asentar otros edificios posteriores (1). En el siglo XIII, tras la reconquista de la ciudad, se inició la construcción sobre los restos del teatro de la zona de la ciudad conocida actualmente como barrio del Pópulo. En ese momento se perdió definitivamente toda referencia al teatro primitivo (2).

El teatro fue descubierto de forma accidental en el año 1980 , tras la demolición de unas naves industriales que lo cubrían parcialmente (Figura 2a). Desde ese momento se ha seguido un proceso sistemático hacia su recuperación. Tras algunas demoliciones de edificios sin ningún interés histórico y posteriores excavaciones (3), en el año 2010 se había recuperado una parte significativa de la cávea del teatro (Figura 2b).

\section{INTERVENCIÓN EN EL ÁREA DEL TEATRO}

\subsection{Descripción de la Intervención}

La intervención para la recuperación del Teatro Romano de Cádiz debía dar solución al dilema de compatibilizar la seguridad y pervivencia de los edificios existentes sobre el teatro con la recuperación de los restos arqueológicos del mismo, de modo que tales zonas de interés cultural quedaran a la luz y pudieran visitarse (Figura 3). La importancia no radicaba sólo en la puesta en valor del teatro, sino también en la correcta conservación de las edificaciones construidas sobre él, puesto que éstas, al formar parte del barrio del Populo, forman parte del patrimonio histórico de la ciudad.

En 2010 se inició una importante intervención que, de forma general, consiste en la generación de una serie de bóvedas bajo los edificios del barrio del Pópulo. Estas bóvedas serán las encargadas de transmitir las cargas a un sustrato de terreno suficientemente resistente (Figura 4a) (4), permitiendo la excavación del terreno bajo ellos y sacando a la luz de esta forma de los restos del teatro. Para la ejecución de las bóvedas se recurrió a la técnica de inyección armada, y más concretamente, a la adaptación de la técnica de los paraguas

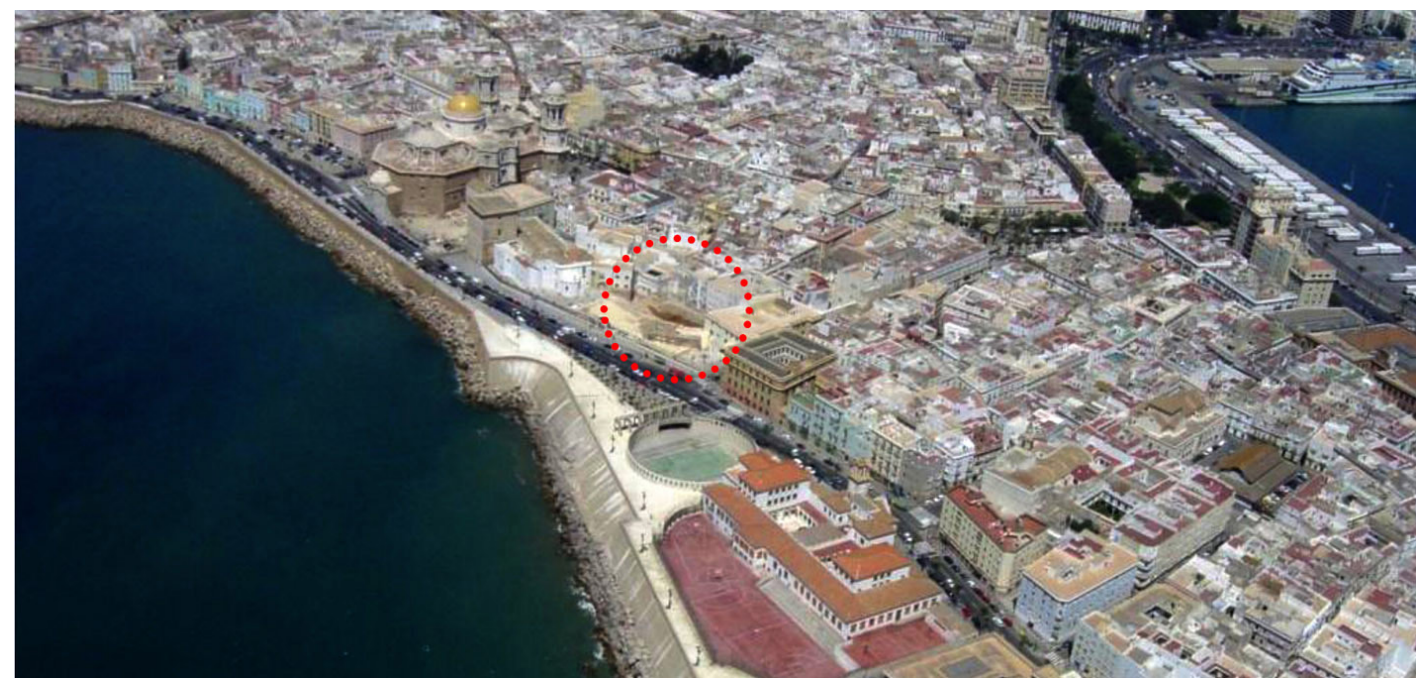

Figura 1. Localización del Teatro Romano de Cádiz.
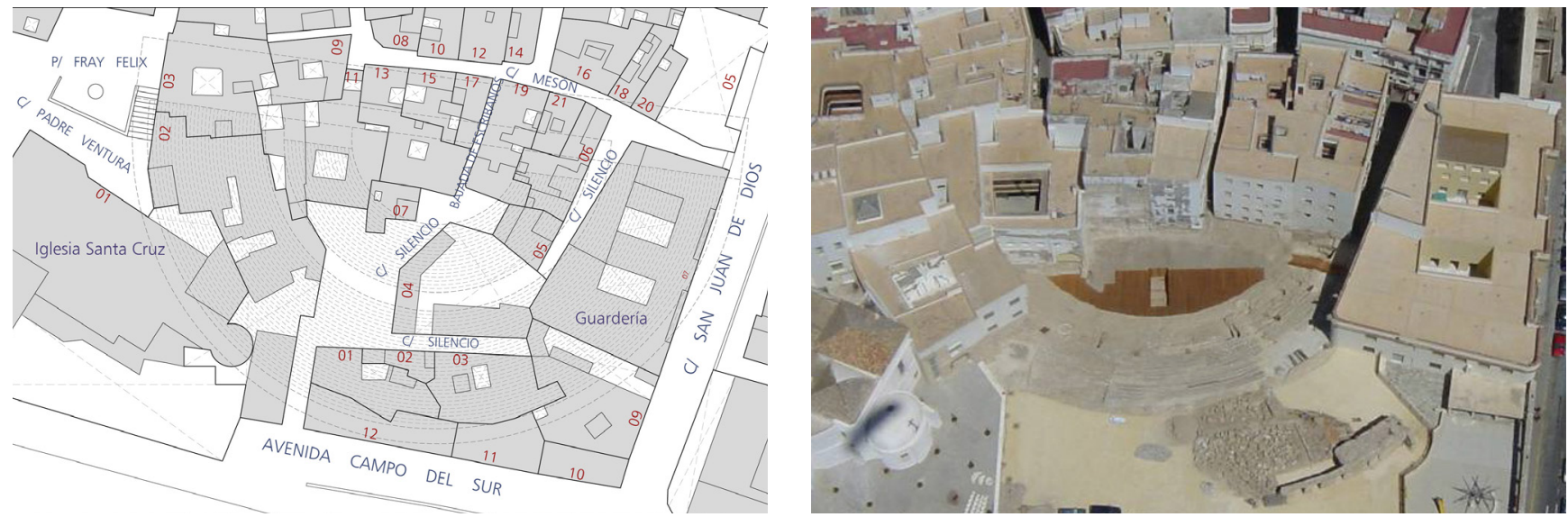

Figura 2. a) Estado del parcelario superpuesto a las trazas del Teatro. Año 1980. b) Estado del Teatro Romano. Año 2010. 


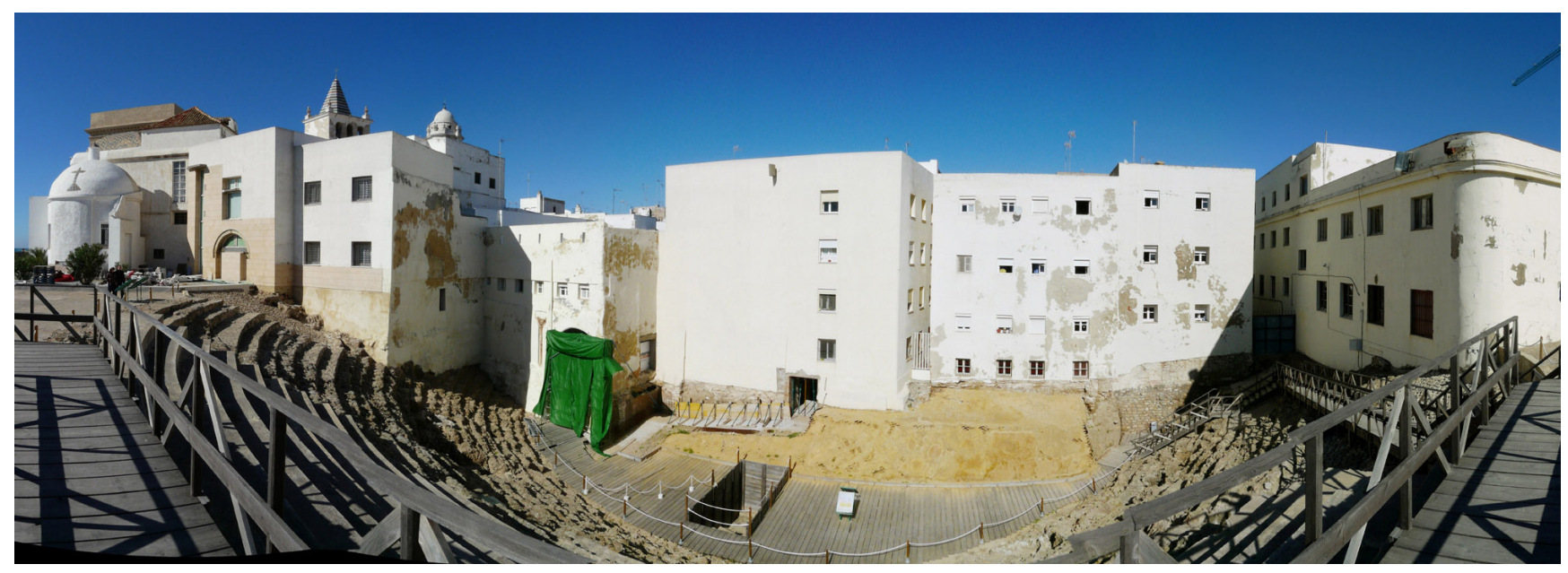

Figura 3. Área del Teatro Romano de Cádiz.

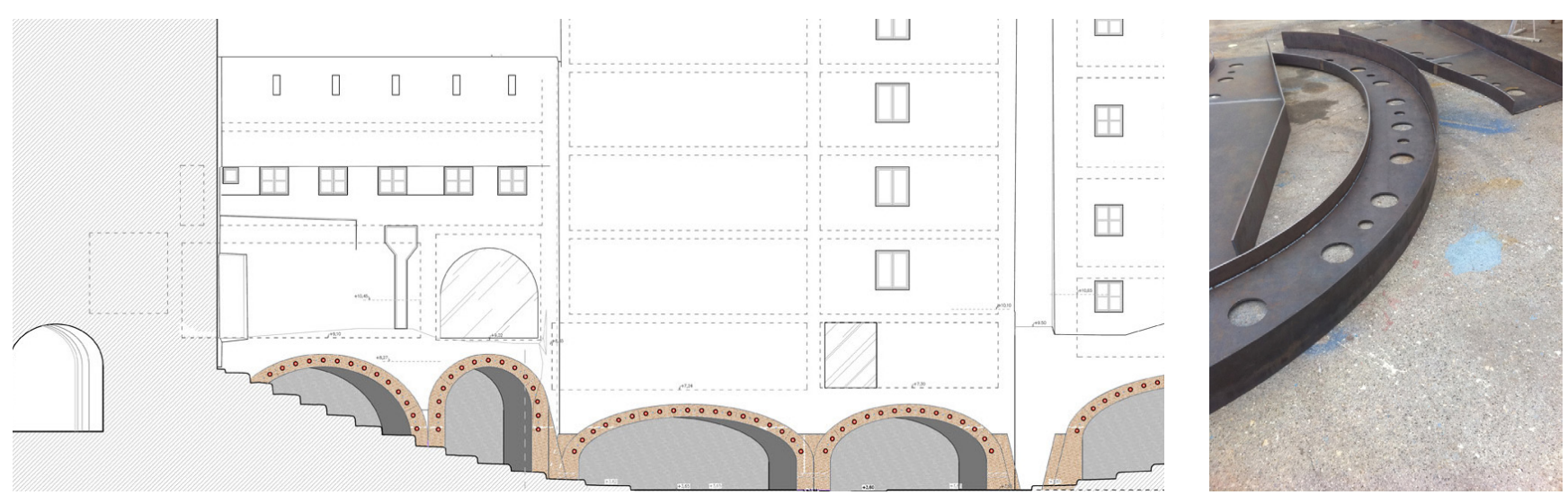

Figura 4. a) Alzado esquemático tras la extracción de tierras. b) Arcos para la ejecución de los paraguas de inyección.

de inyección usada en el campo de la ingeniería para la excavación de túneles (5) (6). Para la generación de las bóvedas, se realizaron inyecciones horizontales con tubos manguito aproximadamente cada $0,50 \mathrm{~m}$, definiendo la posición exacta mediante arcos de acero (Figura 4b). Las cimentaciones y el tramo inferior de los muros de carga se inyectaron verticalmente también usando tubos manguito (7). Todas las lechadas empleadas fueron de cemento-bentonita. El resultado final tras la excavación bajo los paraguas de inyección será un nuevo espacio abovedado debajo de los edificios desde el cual se podrán contemplar los restos del teatro (Figura 5).

\subsection{Control de la Intervención}

El control de la intervención que se está ejecutando sobre el área del Teatro Romano de Cádiz se realiza fundamentalmente por medio de dos técnicas. Éstas son el control topográfico de precisión y el Análisis Modal Operacional.

\section{Control Topográfico de alta precisión}

La técnica de control topográfico fue utilizada durante el proceso de inyección de las bóvedas de hormigón para detectar movimientos que pudieran afectar a la estabilidad estructural de las edificaciones. Se implantó un sistema de lectura en tiempo real mediante la disposición de una estación total robotizada de alto rendimiento con precisión de $0,05 \mathrm{~mm}$ (Figura 6). Se optó por realizar lecturas topográficas en las tres direcciones del espacio mediante la instrumentación de

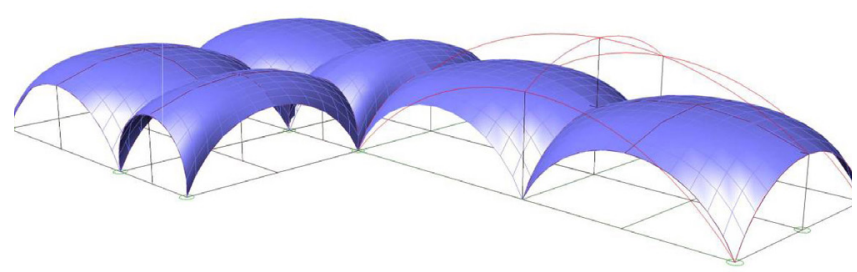

Figura 5. Perspectivas esquemáticas del revestimiento interior de las bóvedas.

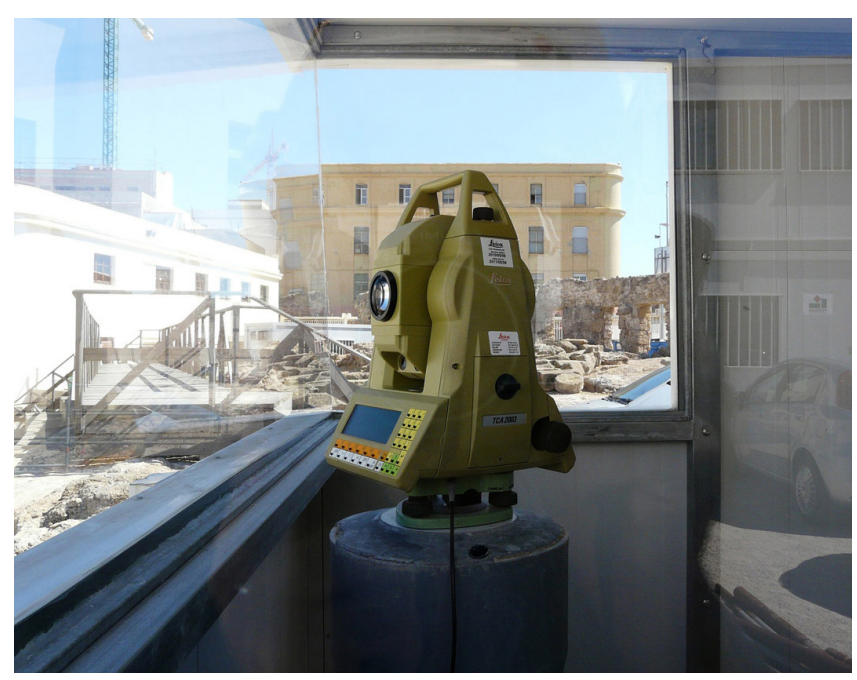

Figura 6. Estación fija de control topográfico. 
veintiséis miniprismas de alta precisión (Figura 7) cuya disposición queda reflejada en la Figura 8. Se dispuso también de una alarma automática con transmisión instantánea a los teléfonos móviles de los técnicos en caso de superación de las deformaciones límites pre-establecidas.

Los resultados recopilados durante la fase de ejecución de los paraguas de inyección indican movimientos insignificantes que no afectan la estabilidad estructural de las edificaciones. Los movimientos registrados entre el 16 de marzo de 2011 y el 27 de marzo de 2012, periodo de ejecución de los paraguas de inyección, presentan en el 99 \% de los casos valores infe-

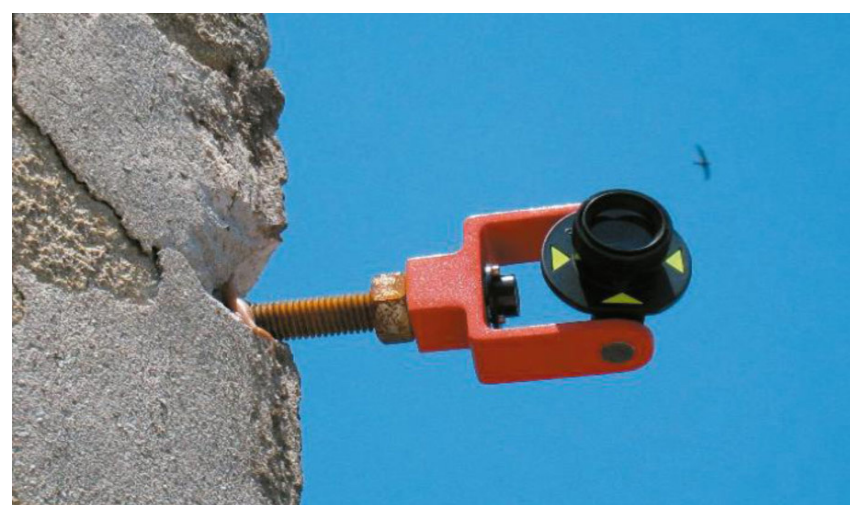

Figura 7. Miniprisma. riores a un milímetro tanto en la dirección vertical como en la horizontal (Figura 9).

\section{Análisis Modal Operacional (OMA)}

Las pruebas de vibración [aambiental están consolidadas desde hace años como uno de los principales métodos experimentales para evaluar el comportamiento dinámico de estructuras a gran escala. La excitación artificial de grandes estructuras conlleva una gran complejidad, lo que hace recurrir al Análisis Modal Operacional (OMA) para la identificación modal de las propiedades dinámicas de la estructura por encima del Análisis Modal Experimental (EMA) (8) (9) (10). En los últimos años son numerosas las aplicaciones de las pruebas de vibración ambiental que podemos encontrar asociadas al mundo de la arquitectura (11). Los buenos resultados obtenidos de estas aplicaciones, el bajo coste económico que supone hacer este tipo de pruebas y la no interrupción del normal funcionamiento del edificio mientras las pruebas son realizadas, son algunos de los motivos que han hecho que éstas sean muy populares. Sin embargo, en el caso concreto de construcciones históricas de fábrica, donde el uso de técnicas no destructivas es un factor fundamental, los casos de aplicación de OMA son menos numerosos debido a dificultades añadidas. Éstas contemplan desde la dificultad que supone realizar una representación exacta de la geometría en este tipo de edificaciones, hasta el complejo comportamiento de los materiales (12) (13) (14) (15) (16).

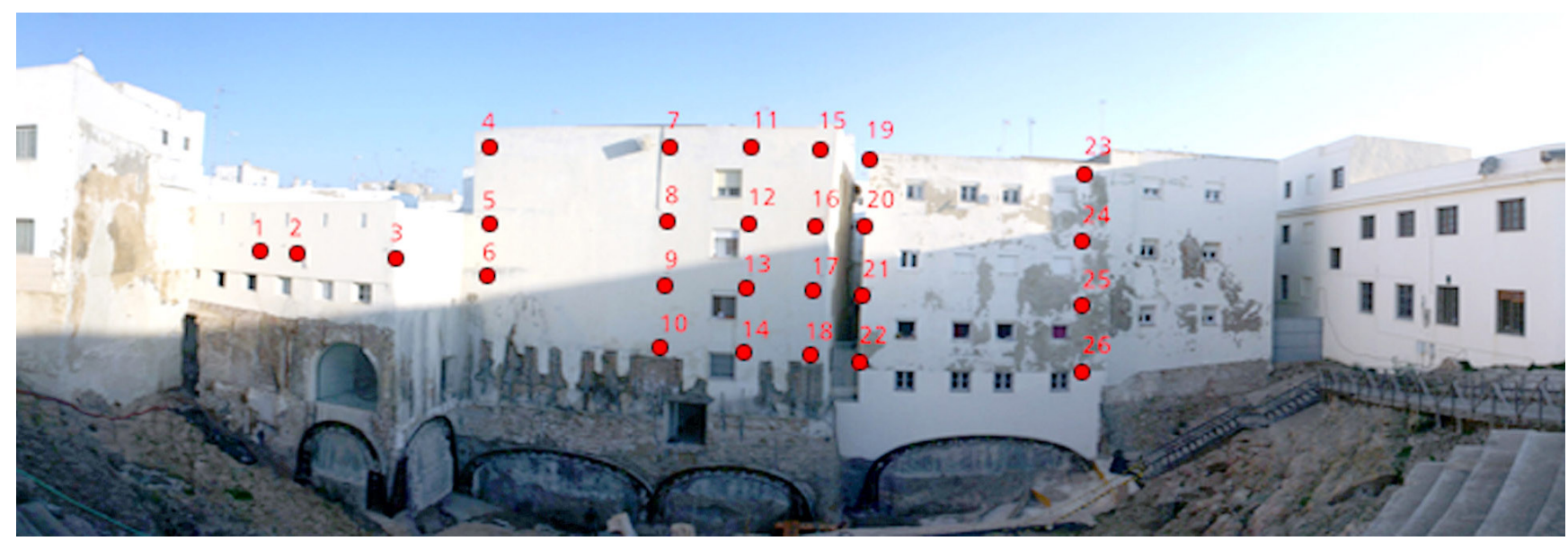

Figura 8. Situación de los miniprismas.


Figura 9. Lecturas de los movimientos verticales y transversales de los puntos 4 a 10 durante la inyección de la bóveda inmediatamente inferior. 


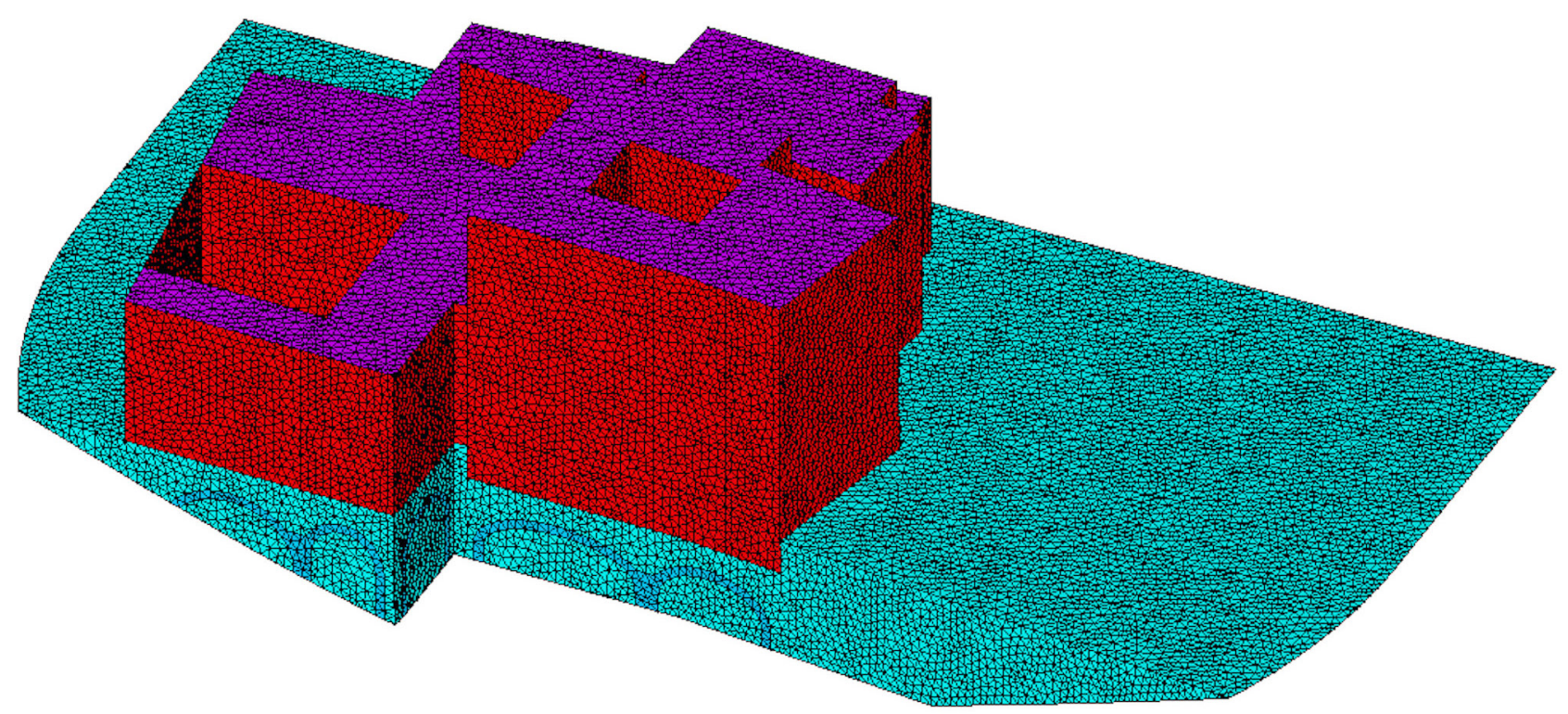

Figura 10. Modelo de EF. Elementos superficiales: SHELL 63. Elementos volumétricos: SOLID 45.

La técnica OMA se ha utilizado en el control de la intervención sobre el Teatro Romano de Cádiz (17) de forma complementaria al control topográfico. La forma de operar consiste en la obtención de los parámetros modales del conjunto estructural antes y después de los trabajos y a través de métodos numéricos y experimentales. La comparación de estos parámetros antes y después de la intervención permite identificar cualquier alteración en el comportamiento estructural del conjunto.

La aplicación del Análisis Modal Operacional requiere de la generación de un modelo de elementos finitos previo. La utilidad del mismo es la identificación de los primeros modos de vibración y, dentro de los mismos, los puntos con mayor desplazamiento modal. De esta forma se decide la posición de los acelerómetros en el ensayo in situ que se llevará a cabo posteriormente (8) (10). El modelo realizado se limitó a la zona de la primera fase de inyección, y se constituye de dos partes fundamentales, los edificios y el terreno. Se modeló utilizando el programa informático ANSYS. Los edificios fueron mallados con elementos superficiales SHELL63, mientras que, para el terreno, se utilizaron elementos sólidos SOLID45. En total resultó un modelo de 975.370 elementos y 189.585 nodos (Figura 10). Las propiedades de los materiales de densidad, módulo de deformación y coeficiente de Poisson para este modelo preliminar se extraen de la bibliografía consultada (18) (19) (20) para posteriormente ser actualizados (Tabla 1).

El cálculo modal realizado permitió identificar los tres primeros modos de vibración (Figura 11) y, por consiguiente, la ubicación de los cuatro acelerómetros de referencia en la cubierta del inmueble monitorizado según se ilustra en la Figura 12.

La monitorización de la estructura y la toma de datos se realizó con un equipo compuesto por ocho acelerómetros uniaxiales de fuerza balanceada Episensor de la casa comercial KINEMETRICS, con ancho de banda de 0-200 Hz, y una central de adquisición de datos tipo GRANITE de la misma marca (Figura 13). El software ARTEMIS se utilizó para el tratamiento e interpretación de los datos recopilados.

Tabla 1. Propiedades mecánicas de los materiales del modelo de EF.

\begin{tabular}{|l|c|c|c|}
\cline { 2 - 4 } \multicolumn{1}{c|}{} & \multicolumn{3}{c|}{ Modelo EF } \\
\cline { 2 - 4 } \multicolumn{1}{c|}{} & $\begin{array}{c}\text { Fábrica } \\
\text { (muros de carga) }\end{array}$ & Terreno & $\begin{array}{c}\text { Hormigón } \\
\text { (forjados) }\end{array}$ \\
\hline Densidad (kg/m m $^{3}$ & 1500 & 1800 & 2500 \\
\hline Módulo de deformación (MPa) & 4000 & 1500 & 21000 \\
\hline Coeficiente de Poisson & 0,2 & 0,2 & 0,2 \\
\hline
\end{tabular}
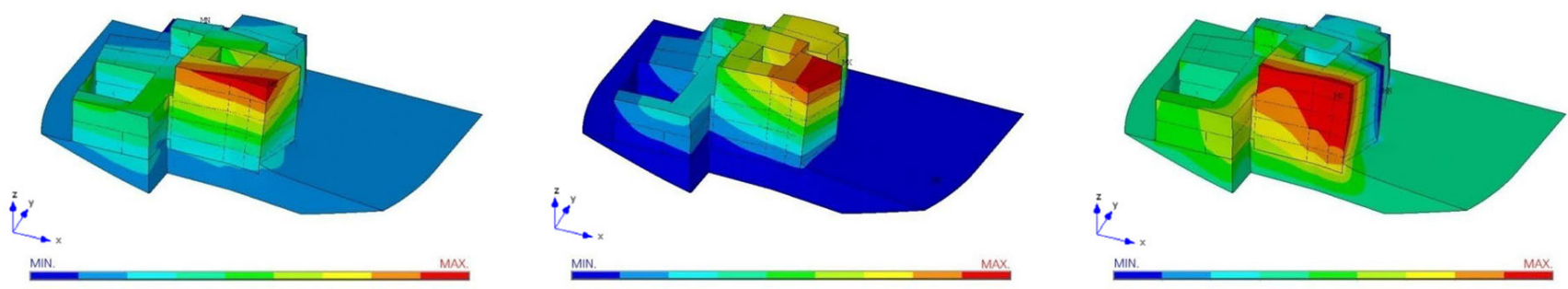

Figura 11. Desplazamientos modales del primer modo de vibración en direcciones X, Y y Z. 


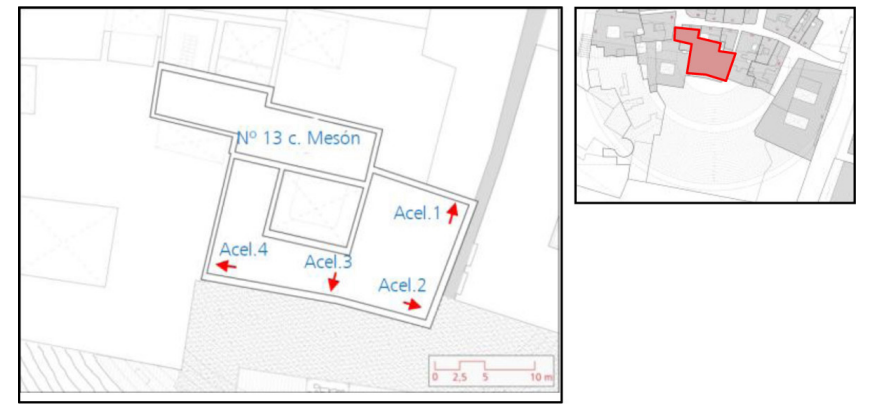

Figura 12. Situación, dirección y sentido de los acelerómetros de referencia.

La primera campaña experimental se realizó antes de comenzar con la intervención. Los acelerómetros de referencia fueron cuatro, situados según el análisis previo (Figura 12). En total se utilizaron ocho acelerómetros, de los cuales cuatro eran de referencia y su posición se ha descrito anteriormente. Los otros cuatro se colocaron siguiendo la vertical de los anteriores en cada una de las plantas, resultando en total cuatro series de medidas con ocho acelerómetros cada una. Cada medida duró quince minutos (17).

Una vez tomados y almacenados los datos, se procedió al tratamiento de los mismos (Figura 14). Se estudió el comportamiento dinámico utilizando dos métodos de identificación modal: el método de Descomposición mejorada en el dominio de la frecuencia (EFFD) y el método de Identificación en el Subespacio Estocástico (SSI), ambos implementados en el programa comercial ARTEMIS.

Con ambos métodos se identificaron tres modos de vibración en un rango de frecuencias de o a $10 \mathrm{~Hz}$. La similitud de los valores de las frecuencias obtenidos por ambos métodos, así como la proximidad del parámetro MAC a 1, avalan la veracidad de los datos obtenidos (Tabla 2). El valor MAC (Modal Assurance Criterion) compara de forma cuantitativa todas las combinaciones posibles de pares de formas modales.

En marzo de 2012, una vez que los paraguas de inyección ya habían sido ejecutados, se llevó a cabo una segunda campaña experimental. La toma y tratamiento de datos siguió el mismo esquema que el desarrollado con anterioridad. Los resultados obtenidos mostraron que tras la intervención en el teatro, el comportamiento dinámico del edificio no se había visto alterado de manera significativa, pues las frecuencias de los tres primeros modos de vibración antes y después de la intervención presentaban diferencias siempre menores al $1 \%$ (Tabla 3).

\section{CONCLUSIONES}

El control topográfico de puntos y el Análisis Modal Operacional (OMA) han sido aplicados en el área del Teatro Romano de Cádiz para controlar la intervención que se está llevando a cabo sobre él. El control topográfico de alta precisión ha sido un proceso continuo de identificación de todo tipo de movimientos de la estructura. En cambio, mediante la técnica OMA,
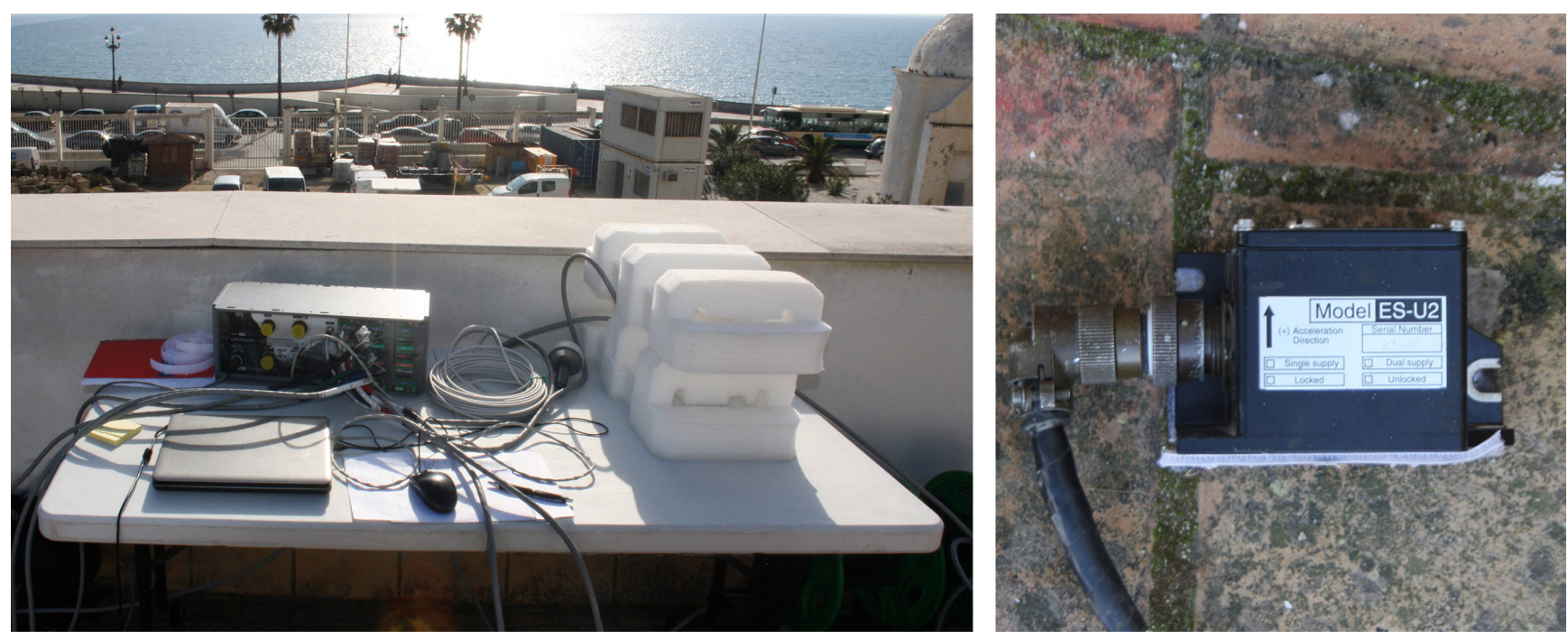

Figura 13. Central de Adquisición de datos, equipo informático y acelerómetro de fuerza balanceada.

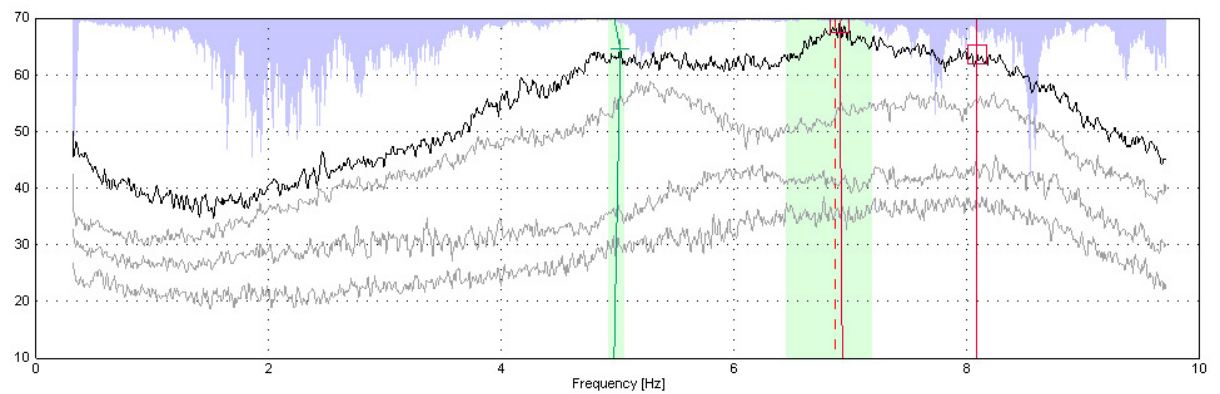

Figura 14. Espectro de respuesta. Identificación de los parámetros modales mediante el método EFDD. 
Tabla 2. Resultados del modelo experimental antes del comienzo de la intervención sobre el área del Teatro Romano de Cádiz.

\begin{tabular}{|l|c|c|c|c|}
\cline { 2 - 4 } \multicolumn{1}{c|}{} & \multicolumn{2}{c|}{ Frecuencia Natural (Hz) } & \multirow{2}{*}{ \% diferencia } & \multirow{2}{*}{ Valor MAC } \\
\cline { 2 - 4 } \multicolumn{1}{c|}{ EFDD } & SSI & 1,24 & 0,96 \\
\hline $\begin{array}{l}\mathbf{1}^{\mathbf{o}} \text { Modo de Vibración } \\
\text { Mov. paralelo eje Y (Figura 10) }\end{array}$ & 4,83 & 4,77 & 0,29 & 0,97 \\
\hline $\begin{array}{l}\mathbf{2}^{\mathbf{o}} \text { Modo de Vibración } \\
\text { Mov. paralelo eje X (Figura 10) }\end{array}$ & 6,88 & 6,86 & 0,85 & 0,95 \\
\hline $\begin{array}{l}\mathbf{3}^{\mathbf{0}} \text { Modo de Vibración } \\
\text { Modo de torsión }\end{array}$ & 8,2 & 8,13 & \\
\hline
\end{tabular}

Tabla 3. Comparación de las frecuencias naturales (Hz) asociadas a los tres modos de vibración.

\begin{tabular}{|l|c|c|c|}
\cline { 2 - 4 } \multicolumn{1}{c|}{} & $\begin{array}{c}\text { F. Natural } \\
\mathbf{1}\end{array}$ & $\begin{array}{c}\text { F. Natural } \\
\text { Modo (Hz) }\end{array}$ & $\begin{array}{c}\text { F. Natural } \\
\mathbf{2} \text { (Modo (Hz) }\end{array}$ \\
\hline Modelo Experimental. Fase Previa. & 4,83 & 6,88 & 8,20 \\
\hline Modelo Experimental. Fase Posterior. & 4,86 & 6,90 & 8,23 \\
\hline \% de diferencia & $0,61 \%$ & $0,28 \%$ & $0,36 \%$ \\
\hline
\end{tabular}

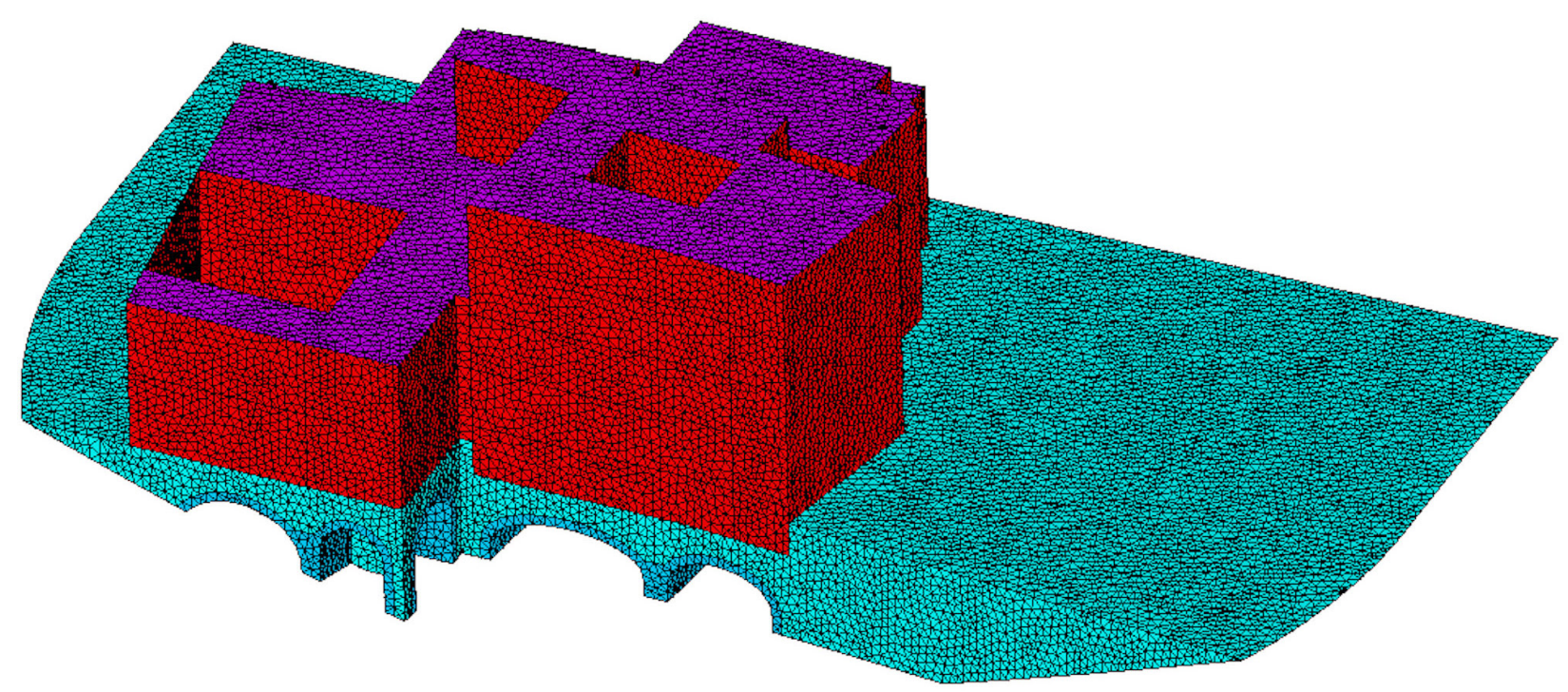

Figura 15. Modelo de EF correspondiente al estado final de la obra.

apoyada en modelos de elementos finitos, se ha evaluado el efecto global de la intervención sobre los edificios, gracias a las pruebas de vibración ambiental realizadas antes y después de la ejecución de los paraguas de inyección. Ambas técnicas son, por tanto, complementarias.

Los resultados recopilados a partir del control de los puntos indican que los movimientos inducidos por la ejecución de los paraguas de inyección son mínimos. En lo que se refiere a la aplicación del Análisis Modal Operacional, una vez comparados los datos relativos a las mediciones experimentales realizadas antes y después de la obra, se concluyó que no había habido importantes modificaciones en la rigidez de la estructura derivadas de la intervención. De la aplicación de las técnicas de control se deriva que la aplicación de la técnica de intervención ha dado buen resultado.
Como continuación de esta investigación, los resultados recopilados están siendo utilizados para validar los modelos de elementos finitos a través del procedimiento conocido con el nombre de model updating. Una vez que el comportamiento dinámico de los modelos de cálculo sea ajustado al comportamiento real del conjunto estructural, estos modelos podrán ser utilizados para evaluar el comportamiento del conjunto en su estado final, así como el efecto de posteriores actuaciones puedan tener sobre el conjunto (Figura 15).

\section{AGRADECIMIENTOS}

Los autores agradecen al proyecto DPI2010-21590-Co2-02 del Ministerio de Economía y Competitividad el apoyo económico prestado.

\section{REFERENCIAS}

(1) Corzo-Sánchez, R. (1993). El Teatro Romano de Cádiz. Teatros Romanos de Hispania. Cuadernos de Arquitectura Romana, 2: 133-140.

(2) Fresnadillo-García, R., Tabales-Rodríguez, M. A., Maya-Torcelly, R., Jurado-Fresnadillo, G., Pajuelo-Sáez, M. (20o8). Cádiz en la edad media. Revista Atlántica-Mediterránea de Prehistoria y Arqueología Social - RAMPAS, 10: 399-411. 
(3) Pérez-Alberich, J. M. (2011). La revitalización del Teatro. De los años ochenta a la actualidad. El Theatrum Balbi de Gades. En Seminario «El Teatro Romano de Gades. Una mirada al futuro», Cádiz 18-19 Noviembre 2009. Cadiz: Servicio de Publicaciones Universidad de Cádiz.

(4) Yanes, E., Cobo, A. (2011). Un proyecto arquitectónico singular de consolidación. La futura mirada al Teatro Romano Gaditano. El Theatrum Balbi de Gades. En Seminario «El Teatro Romano de Gades. Una mirada al futuro», Cádiz 1819 Noviembre 2009. Cadiz: Servicio de Publicaciones Universidad de Cádiz.

(5) Da Casa, F., Echeverría, E., Celis, F. (2007). La intervención bajo rasante, la importancia de su conocimiento. La técnica de la inyección armada. Informes de la Construcción, 59(505): 21-35, doi: http://dx.doi.org/10.3989/ic.2007.v59. i505.498.

(6) Coulter, S., Martin, C. D. (2006). Single fluid jet-grout strenght and deformation properties. Tunnelling and Underground Space Technology, 21(6): 690-695, doi: http://dx.doi.org/10.1016/j.tust.2005.12.136.

(7) Rodríguez-Mayorga, E., Yanes, E., Compán, V., Sáez-Pérez, A. (2013). La restauración del templo parroquial de San Dionisio (Jerez de la Frontera, España). La inyección como método de reparación de estructuras de fábrica. Informes de la Construcción, 65(529): 5-16, doi: http://dx.doi.org/10.3989/ic.11.130.

(8) Ramos, L. (2007). Damage Identification on Masonry Structures Based on Vibration Signatures (Tesis doctoral). University of Minho .

(9) Jiménez, J. F. (2010). Estudio del comportamiento dinámico de pasarelas peatonales esbeltas bajo sobrecarga de uso (Trabajo de investigación para la obtención del diploma de estudios avanzados - DEA). Sevilla: Departamento de Mecánica de Medios Continuos, Teoría de Estructuras e Ingeniería del Terreno - Universidad de Sevilla.

(10) Rodrigues, J. (2004). Identificação Modal Estocástica, Métodos de Análise e Aplicações em Estruturas de Engenharia Civil (Tesis doctoral). University of Porto.

(11) González, M. N., Cobo, A., Fuente, J.V. (2013). Obtención de modelos de cálculo de sistemas provisionales de protección de borde mediante la técnica de Análisis Modal Operacional. Informes de la Construcción, 65(529): 99-106, doi: http:// dx.doi.org/10.3989/ic.11.133.

(12) Ramos, L. F., Aguilar, R., Lourenço, P., Moreira, S. (2012). Dynamic Structural Health Monitoring of Saint Torcato Church. Mechanical Systems and Signal Processing, 35(1-2): 1-15, doi: http://dx.doi.org/10.1016/j.ymssp.2012.09.007.

(13) Votsis, R. A., Kyriakides, N., Chrysostomou, C., Tantele, E., Demetriou, T. (2012). Ambient Vibration Testing of Two Masonry Monuments in Cyprus. Soil Dynamics and Earthquake Engineering, 43: 58-68, doi: http://dx.doi.org/10.1016/j. soildyn.2012.07.015.

(14) Gentile, C., Saisi, A. (2004). Dynamic-based F.E. Model Updating to Evaluate Damage in Masonry Towers. En Proceedings of the 4th International Seminar on Structural analysis of Historical Constructions, Vol. 1: 439-449. Padova, Italy.

(15) Ramos, L. F., Marques, L., Lourenço, P., De Roeck, P., Campos-Costa, A., Roque, J. (2010). Monitoring Historical Masonry Structures with Operational Modal Analysis: Two Case Studies. Mechanical Systems and Signal Processing, 24(5): 1291-1305, doi: http://dx.doi.org/10.1016/j.ymssp.2010.01.011.

(16) Foti, D., Diaferio, M., Giannoccaro, N., Mongelli, M. (2012). Ambient Vibration Testing, Dynamic Identification and Model Updating of a Historic Tower. NDT and International, 47: 88-95, doi: http://dx.doi.org/10.1016/j.ndteint.2011.11.009.

(17) Pachón, P. (2012). Predicción de la estabilidad estructural mediante análisis modales operacionales (Trabajo de investigación para la obtención del Máster de Innovación en Arquitectura: Tecnología y Desarrollo). Sevilla: Departamento de Mecánica de Medios Continuos, Teoría de Estructuras e Ingeniería del Terreno - Universidad de Sevilla.

(18) Ministerio de Fomento. (2008). Norma EHE Instrucción de Hormigón Estructural. http://www.fomento.es/MFOM/ LANG_CASTELLANO/ORGANOS_COLEGIADOS/CPH/instrucciones/EHE_es/.

(19) Augenti, N., Parisi, F., Acconcia, E. (2012). MADA: online experimental database for mechanical modeling of existing masonry assemblages. En Proc., 15th World Conference on Earthquake Engineering, Lisbon, Portugal.

(20) CTE. (2006). Código Técnico de la Edificación. http://www.codigotecnico.org/web/recursos/documentos/. 